\title{
Lepromin as an indicator and inducer of protective immunity
}

\section{MJ LEFFORD}

Department of Immunology and Microbiology, Wayne State University School of Medicine, Detroit, MI 48201, U.S.A.

Let us first consider the nature of lepromin. Lepromin is prepared by excising superficial lepromata from patients with lepromatous leprosy. The leproma proper is dissected from the attached normal tissues and autoclaved. The cooked leproma is then ground up in a sterile saline or buffer and passed through a coarse filter. Phenol is added as a preservative and the material is distributed into vials and re-autoclaved. In effect, lepromin is a twice-cooked soup, or more correctly, consommé, of human inflammatory tissue and leprosy bacilli. How is so crude a product standardized? Amazing as it may seem, until the last 20 years or so, the basis of standardization was the weight of leproma, regardless of its content of leprosy bacilli $(1,2)$. Now, lepromin is adjusted to contain $1.6 \times 10^{8}$ acidfast bacilli/ml (3). This method of standardization leaves much to be desired for two reasons. The first is the tendency of leprosy bacilli to stick together forming clumps whose content of single bacilli can be estimated only crudely. The second problem is that leprosy bacilli are notoriously difficult to stain, and a variable proportion of organisms are chromophobic: they are not stained by the commonly-used methods.

When bjological products are used for human diagnostic testing it is usually mandatory that their functional activity be assayed in experimental animals. Such is the practice, for example, in standardizing tuberculin purified derivative (PPD). No such requirement applies to lepromin.

The availability of the source material, human lepromata, is scarce, variable and unpredictable in its content of bacilli. Also, the amount of human tissue contamination may vary from batch to batch. This is not a trivial consideration, since there are published reports of lepromin reactions being induced by normal human tissue preparations (4). Moreover, the production of lepromin is essentially a cottage industry, performed by those with access to the source material, rather than by people with expertise in the preparation of pharmaceutical biologicals. Consequently, although most preparers follow the same general methodology, local variations in the technical procedures doubtless exist. Given these variables, it is remarkable that lepromin has proved to be so useful a product. How- 
ever, in comparing published studies one should bear in mind that variations in the lepromin used may account, at least in part, for otherwise inexplicable discrepancies.

In recent years an additional source of lepromin has become available, namely, that obtained from armadillos (Dasypus novemcinctus) infected artificially with $M_{0}$ leprae from human or mouse passage sources. There are potential advantages to the use of this material and some disadvantages. The main advantage is that a relatively large bulk of tissue from a single armadillo or pooled from several armadillos, can be frozen, and later dispatched to a central laboratory, where a highly standardized procedure can be applied to the manufacture of large batches of material. These can be assayed at leisure and then distributed world-wide, ensuring that numerous investigators are working with the same material, so favoring comparability of data. of the several disadvantages, one is that armadillo lepromin may differ antigenically from human lepromin, in which case results using the newer material may be incompatible with the large body of evidence derived from human lepromin usage. A second problem is that the armadillo lepromin may be contaminated with other microbes or microbial products and will certainly be contaminated with armadillo antigens. Before becoming unduly alarmed by these considerations, one should realize that similar risks apply to human lepromin. The human material may also express transplantation antigens to which some subjects may respond, particularly if lepromin tests are administered repeated1y. The available data suggest that armadillo and human lepromins elicit comparable responses in man and experimental animals; and microbial and tissue contamination does not appear to be a material problem $(5,6)$.

There are two types of response to lepromin: an early response, the Fernandez reaction, which occurs 24-48 hours after injection; and the late, Mitsuda reaction which is usually read one month later. Criteria of positivity (doubtful, moderate or strong) have been designated for both types of reaction $(1,2)$ based on the diameter of induration at the reaction site.

Such classification is helpful to the clinician in assisting him to attach a label to a patient. The scientific validity of such classification is more questionable. For example, one patient with a Mitsuda reaction of $5 \mathrm{~mm}$ diameter is designated 'weak positive', while one with a $6 \mathrm{~mm}$ lesion is designated 'moderate positive'. Given the difficulty and observer variation in making these measurements accurately to the nearest $\mathrm{mm}$, the separation of these patients into separate categories of positivity is more misleading than helpful. Moreover, the measurement of a lump in the skin tells one very little about the nature of that lump. Thus, very large doses of lepromin may even induce positive Mitsuda reactions in lepromatous leprosy patients (5). Such reactions are not hypersensivity granulomas but are nonspecific inflammatory reactions of the foreign body type (7). Because of this and other problems investigators have sought confirmatory evidence that a positive Mitsuda reaction really is positive. One approach has been to biopsy the reaction site to see 
whether the injected bacilli have been cleared from the reaction site: clearance indicating positivity. Another alternative is to restrict positivity only to those reactions which ulcerate (8), a course that would drastically reduce the number of Mitsuda-positive normal subjects.

There exists a mountain of data on the use of the lepromin test in the diagnosis of leprosy, and the general conclusion is that the test is of no value for this purpose. The Fernandez reaction is usually negative in normal populations in nonendemic areas, but is also frequently negative in leprosy patients. On the other hand, the Mitsuda reaction is positive in both normal subjects and many leprosy patients. The value of the lepromin reaction lies in the classification of established leprosy. In general, as one passes through the clinico-pathological spectrum of leprosy from polar tuberculoid (TT) to polar lepromatous (LL) there is a progressive loss of CMI to M. leprae, however expressed: Fernandez reactivity, Mitsuda reactivity, or in vitro lymphocyte transformation (LT) to specific M. leprae antigens (9).

Before going further, let me first dismiss the hypothesis that the lack of lepromin reactivity in LL patients is caused by nonspecific immunodepression. Although generalized deficiencies of CMI have been demonstrated in LL by some investigators, others have been unable to confirm those results. Moreover, significant immunodeficiency in LL offends against clinical common sense. DO LL patients present with an immunodeficiency syndrome, as do patients with certain lymphomas, congenital abnormalities of the immune system, or those receiving immunosuppressive drugs? No. Where nonspecific immunodeficiency exists, it is probably a consequence of LL rather than its cause. However, the most remarkable evidence of the specificity of the non-reactivity to lepromin is that LL patients may respond to other mycobacterial antigens. Such patients may give a DTH reaction in response to human purified protein derivative (PPD) and a Mitsudatype response to BCG vaccine (10). Such exquisite specificity is remarkable because of the known cross-reactivity between the antigens from different mycobacterial species, as measured by CMI and humoral immunity. With respect to CMI, there is abundant experimental evidence of cross-reactivity between mycobacterial species with respect to protective immunity, DTH and LT. Similarly, analysis of serologicallydetermined mycobacterial antigens have demonstrated widespread interspecies distribution of some antigens, and there is no really strong evidence that a single species specific antigen exists for any mycobacterial species, including M. leprae (11). Yet the human $\mathrm{T}$ cell can distinguish between the antigens of M. leprae and BCG.

Let me now turn to the interpretation of the lepromin test, and to a lesser extent, the LT test. There are basic immunological requirements for each of these tests.

1. The antigens must first be processed by macrophages.

2. There must exist clones of $T$ lymphocytes bearing recognition sites for the relevant mycobacterial antigens.

3. There must be a mechanism for bringing the macrophage-processed antigen into close physical association with the appropriate $\mathrm{T}$ 
cells in vivo.

4. Suppressor mechanisms must be held in abeyance.

I will discuss these factors in relation to DTH (the Fernandez reaction), first.

Macrophages are so widely distributed in the body that one cannot imagine these cells being a limiting factor, except where there is generalized bone marrow insufficiency. (In that case, leprosy might be the least of the patient's worries). However, although many if not all, macrophages can process antigen, fewer can 'present' antigen to T cells. For this purpose a subpopulation of macrophages is required that bears surface antigens specified by the immune response region of the major histocompatibility complex (12). Such macrophages may be limiting at a particular site in a leprosy patient. of particular relevance are the Langerhans cells of the skin, which may be essential for dermal CMI reactivity (13). Whether or not these cells are altered numerically or functionally in leprosy, I do not know.

An absence of the appropriate $\mathrm{T}$ cells is conceivable but improbable, as a consequence of congenital deletion of the genes coding for the $\mathrm{T}$ cell clones that recognize $\mathrm{M}$. leprae antigens. Such a deletion might well occur for a single antigenic determinant, but lepromin contains many antigens each of which probably bears several determinants, so it is most improbable that all the corresponding genes are deleted.

The problem of bringing together the lymphocytes and macrophages in the skin reaction site is often forgotten, particularly by those who perform their DTH tests in vitro (LT or lymphokine assays). It appears that lymphocytes enter inflammatory reactions in an immunological non-specific fashion; that is, there is no preferential migration of $\mathrm{M}$. leprae-sensitized lymphocytes into a lepromin reaction site (14). There is yet a further, and important, consideration. Not all circulating $\mathrm{T}$ cells have the ability to enter reaction sites. The majority of $\mathrm{T}$ cells that recirculate from blood to lymph are longlived. In tuberculosis, protective immunity is vested predominantly in these cells, yet, paradoxically, these cells cannot enter DTH inflammatory reaction sites analogous to the Fernandez reaction. However, a numerically much smaller population of short-lived $\mathrm{T}$ cells can do so. The latter cells are either actively proliferating in response to antigen, or the immediate progeny of proliferating cells. In brief, a relatively small subset of $\mathrm{T}$ lymphocytes can enter DTH reactions, and of those that enter, a small proportion can recognize the eliciting antigens. Consequently, a positive reaction is contingent on the presence of substantial numbers of specific DTH effector lymphocytes in the circulation at the time of antigen injection. This will occur when there is active lymphoproliferation of specific clones in vivo. Such proliferation occurs in response to antigen release, a situation which prevails in reversal reactions and during chemotherapy. In fact, spontaneous DTH reactivity may be a component of reversal reactions (15). There is some evidence that the T cells that participate in LT responses are also short-lived, newly-formed cells. Accordingly, one would expect high LT responses during re- 
versal reactions, and close statistical associations between LT responsiveness and Fernandez reactivity. I do not propose to discuss suppressor mechanisms, but anything that interrupts the described chain of cellular reactions will automatically suppress DTH.

With some understanding of the Fernandez reaction, let us consider the Mitsuda reaction. Both reactions are contingent upon macrophage processing and presentation of antigen, sensitized $\mathrm{T}$ cells, and interaction between them. So why is the Fernandez reaction less common, even among leprosy patients, than the Mitsuda reaction, which is seen in all forms of leprosy but LL, and also among healthy individuals? One critical factor is time. The host has only 48 hours in which to generate the Fernandez reaction, an impossible task unless the appropriate $\mathrm{T}$ cells are already circulating in substantial numbers. By contrast, a more leisurely tempo prevails in the Mitsuda test. Consider the following scenario. The lepromin antigens are processed in the. skin, and regardless of whether or not a Fernandez reaction ensues, those antigen-presenting macrophages travel to the draining lymph nodes and wander into the paracortex where they mingle with the aforementioned long-lived, recirculating, small lymphocytes that migrate through lymph nodes. Interaction between the macrophages and specific clones of $\mathrm{T}$ lymphocytes occurs and the latter proliferate. The peak proliferative response may take one to several weeks to develop depending on the dose of antigen, but then a population of newly-formed, short-lived cells leaves the lymph nodes, enters the circulation and is available to enter the reaction site, belatedly. An immunologically specific inflammatory reaction ensues resulting in formation of a hypersensitivity granuloma.

It follows from the above description that a normal subject unexposed to M. leprae or cross-reactive material cannot develop a Fernandez reaction, but may develop a Mitsuda reaction, which acts as an immunizing dose of $M$. leprae vaccine. In actuality, this is such an important issue that I will restate it. A positive Fernandez reaction is a measure of high CMI reactivity to Meprae resulting from active or cross-reactive infection; The Mitsuda reaction is a measure of the ability to generate a CMI response to an immunizing dose of M. leprae. If the subject is infected with M. leprae, the Mitsuda response may be highly accelerated and evident within a few days, due to the preexistence of $M$. leprae-sensitized memory $T$ cells, which are not themselves effector lymphocytes.

The burning question is what relation does the lepromin test bear to the ability of the host to resist Meprae infection? In general, both the Fernandez and Mitsuda reactions indicate a state of specific CMI, the effector lymphocytes prevailing over suppressor factors, a corollary of which is enchanced resistance to Meprae infection. Certainly, in rats immunized with BCG, ahymphoid cell population that mediates DTH also mediates protective immunity $(16,17)$.

There is a current tendency to regard Fernandez positivity and LT positivity as poor correlates of immunity and Mitsuda positivity as a good correlate $(18,19)$. It might be useful to reflect upon this 
situation.

In tuberculosis, there is a strong correlation between the tuberculin hypersensitivity that accompanies subclinical tuberculosis and subsequent resistance to reinfection. Yet apparently normal subjects with florid tuberculin reactivity appear to be hypersusceptible to tuberculosis. In other words, moderate tuberculin hypersensitivity is protective, whereas very high hypersensitivity increases the risk of disease (20). I would suggest that the same may be true in leprosy.

How is one to explain such observations? I believe that the answer lies in suggestions that there may be more than one type of delayed hypersensitivity reaction (21). One type of reaction is associated with protective immunity, while another is not. I suspect that the florid type of $\mathrm{DTH}$ reaction that is associated with hypersusceptibility, is qualitatively different from that type of DTH associated with protective immunity.

LT responses are also more complex than they first appear. It is commonly assumed that LT is a measure of proliferation of $\mathrm{T}_{\mathrm{DTH}} \mathrm{lym}_{\mathrm{Y}}$ phocytes, and so it is, usually. However, T helper cells can also participate in these responses (22), as can B cells (23). The latter may proliferate in a T-dependent fashion, that is, secondary to $\mathrm{T}$ cell proliferation; but $B$ cells can also respond directly to certain mitogens, one of which is tuberculin PPD (24). It is quite possible that $\mathrm{M}_{0}$ leprae antigens may also act as $\mathrm{B}$ cell mitogens. These problems are in urgens need of clarification if we are to fully understand the relationship between DTH, LT and protective immunity.

Finally, I wish to discuss the interpretation of the lepromin reaction in relation to leprosy immunization. It seems probable that the leprosy vaccine will consist of irradiation - sterilized M. leprae grown in armadillos. In effect, the vaccine will be closely analogous to standard lepromin. As such the vaccine may elicit lepromin reactions of the Fernandez and Mitsuda types. Positive Fernandez and accelerated Mitsuda reactions signify previous exposure to $M_{0}$ leprae, or related mycobacteria, and need not be considered further. The real problem concerns the reactions in those not previously sensitized to mycobacterial antigens. These subjects would not develop Fernandez reactions at all, and the incidence of Mitsuda reactions will probably be similar to that found in leprosy non-endemic areas, with most people giving positive reactions. The negative reaction subjects would appear to be un-immunized, but are they un-immunizable? I would suggest not. It is more probable that virtually everyone is immunizable, but the standard dose of vaccine (whatever that happens to be) may be insufficient to immunize the low responder segment of the population (25). A proportion of nonresponders to primary immunization should not surprise us. Dead mycobacteria other than $M$. leprae are notoriously poor immunogens unless used with adjuvants such as mineral oil. For example, to dependably immunize mice with heat-kilg ed BCG a dose of 1-4 mg bacilli is necessary, equivalent to $1-4 \times 10^{2}$ bacilli (26). This is a 100-fold higher dose of organisms than is administered in a standard lepromin test, and 10-40 fold 
higher than the dose of irradiated M. leprae that effectively immunizes some strains of mice $(27,28)$. $\overline{\mathrm{Dr} . \mathrm{P} .} \mathrm{J}$. Patel and I have discovered that mouse strains vary in their responsiveness to irradiated M. leprae, ang some mice respond very poorly to a dose of 100 ug, equivalent to $10^{\circ}$ bacilli. Similar low responsiveness in man may be overcome by several maneuvers: several sequential injections of the standard dose of vaccine, a single repeated vaccination using a larger dose of vaccine, or admixture of living BCG and dead in. leprae vaccines (29). Any residual subjects that do not respond to any immunizing regimen may be presumed to be at high risk of infection.

What of those immunized people who develop a positive Mitsuda reaction; can one be confident that they have been rendered resistant

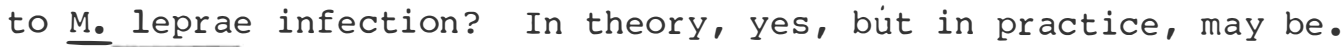
Even the most impressively successful BCG vaccine trial showed that protection was not absolute and waned with time (20). It would be foolish to expect to do better than that. One might speculate whether it is possible to identify those individuals, who though apparently immunized, are least resistant to M. leprae. I would guess that such individuals may be among a population of excessively high responders. This notion is based on observations of mouse strains infected with . lepraemurium. One strain of mice develops a very good CMI response to infection, yet cannot control progressive infection, due to its sensitivity to suppressive influences $(30,31)$. On the other hand, a resistant strain of mice developed CMI more slowly, but the resulting immunity was stable and less easily suppressed.

\section{References}

1 WHO Expert Committee on Leprosy. First report. Wld Hlth Org Techn Rep Ser, 1951, No 71.

2 cochrane RG, Davey $\mathrm{TF}$, eds. Leprosy in Theory and Practice, 2nd ed. Baltimore: Williams and Wilkins Co, 1964, 188.

3 Abe M, Nakayama T, Yanagisawa K, Maeda M, Asami N, Tachikawa N, Okamura K. Studies on the preparation, standardization and preservation of lepromin - comparative experiments with lepromin made by the Mitsuda - Hayashi's original or the Wade's improved method and with a lepromin preserved by freeze-drying "in vacuo". La Lepro, 1961, 30, 163-68.

4 Hart PD, Rees RJW. Lepromin an $\bar{d}$ Kveim antigen reactivity in man, and their relation to tuberculin reactivity. Brit Med Bull, $1967,23,80-85$.

5 Bechelli LM, Haddad W, Pagnano PMG, Neves RG, Melchior E, Fregnan RC. Double blind trial to determine the late reactivity of leprosy patients and unaffected persons to different concentrations of armadillo lepromin in comparison to human lepromin. Int J Lepr, 1980, 48, 126-134.

6 Myrvang B, Godal T, Ridley DS, Frøland SS, Song YK. Immune responsiveness to Mycobacterium leprae and other mycobacterial 
antigens throughout the clinical and histopathological spectrum of leprosy. Clin Exp Immunol, 1973, 14, 541-553.

7 Convit J, Avila JL, Goihman-Yahr M, Pinardi ME. A test for the determination of competency in clearing bacilli in leprosy patients. Bull wld Hlth Org, 1972, 46, 821-826.

8 walter J, Tamondong GT, Garbajosa PG, Bechelli LM, Sansarricq H, Lwin K, Gyi MM. Ncte on some observations about the postlepromin scar. Lepr Rev, 1977, 48, 169-174.

9 Godal T, Myrvang B, Stanford JL, Samuel DR. Recent advances in the immunology of leprosy with special reference to new approaches in immunoprophylaxis. Bull Inst Past, 1974, 72, 273-310.

10 Convit J, Aranzazu N, Pinardi M, Ulrich M. Immunological changes observed in indeterminate and lepromatous leprosy patients and Mitsuda-negative contacts after the inoculation of a mixture of Mycobacterium leprae and BCG. Clin Exp Immunol, 1979, 36, $214-220$.

11 Harboe M, Closs O, Bjorvatn B, Kronvall G, Axelsen NH. Antibody response in rabbits to immunization with Mycobacterium leprae. Infect Immun, 1977, 18, 792-805.

12 Rosenthal AS, Shevach $\overline{\mathrm{EM}}$. Function of macrophages in antigen recognition by guinea pig $\mathrm{T}$ lymphocytes. I. Requirement for histocompatible macrophages and lymphocytes. J Exp Med, 1973, $138,1194-1212$.

13 Silberberg-Sinakin I, Baer RL, Thorbecke GJ. Langerhans cells. Prog Allergy, 1978, 24, 268-294.

14 McGregor DD, Logie PS. The mediator of cellular immunity. VII. Localization of sensitized lymphocytes in inflammatory exudates. J Exp Med, 1974, 139, 1415-1430.

15 Bjune G, Barnetson RS, Ridley $\overline{\mathrm{DS}}$, Kronvall G. Lymphocyte transformation test in leprosy; correlation of the response with inflammation of lesions. Clin Exp Immunol, 1976, 25, 85-94.

16 Lefford MJ. Properties of peritoneal exudate lymphocytes that mediate tuberculin delayed-type hypersensitivity and antituberculosis immunity. I. The effect of cytotoxic drugs. Immunology, 1980, 41, 635-642.

17 Lefford MJ. Properties of peritoneal exudate lymphocytes that mediate tuberculin delayed-type hypersinsitivity and antituberculosis immunity. II. Velocity sedimentation analysis. Immunology, 1980, 41, 643-651.

18 Bjune G. Variation of in vitro lymphocyte responses to M. leprae antigen in borderline tuberculoid leprosy patients. Int J Lepr, 1980, 48, 30-40.

19 Goihmann-Yahr $\bar{M}$. Thoughts on the immunology of leprosy. Int J Lepr, 1980, 48, 435-439.

20 Fourth Report to the Medical Research Council by its Tuberculosis Vaccines Clinical Trials Committee. Bull Wld Hlth Org, 1972, $46,371-385$. 
21 Rook GAW. Three forms of delayed skin-test response evoked by mycobacteria. Nature (Lond), 1978, 271, 64-65.

22 Corradin G, Etlinger HM, Chiller JM. Lymphocyte specificity to protein antigens. I. Characterization of the antigen-induced in vitro $\mathrm{T}$ cell-dependent proliferative response with lymph node cells from primed mice. J Immunol, 1977, 119, 1048-1053.

23 Osborne DP, Katz DH. Antigen-induced deoxyribonucleic acid synthesis in mouse lymphocytes. II. Analysis of the cell populations required for and responsing to antigen stimulation in vitro. J Immunol, 1973, 111, 1176-1182.

24 Sultzer BM, Nilsson BS. PPD tuberculin - a B-cell mitogen. Nature New Biol, 1972, 240, 198-200.

25 McDevitt HO, Benacerraf B. Genetic control of specific immune responses. Adv Immunol, 1969, 11, 31-74.

26 Weiss DW, WElls AQ. Immunization with dead tubercle bacilli. Tubercle (Lond), 1956, 37, 137-140.

27 Patel PJ, Lefford MJ. Induction of cell-mediated immunity to Mycobacterium leprae in mice. Infect Immun, 1978, 19, 87-93.

28 Patel PJ, Lefford MJ. Specific and nonspecific resistance in mice immunized with irradiated Mycobacterium leprae. Infect Immun, 1978, 20, 692-697.

29 Convit J, Ulrich M, Aranzazu N. Vaccination in leprosy - observations and interpretations. Int J Lepr, 1980, 48, 62-65.

30 Lefford MJ, Patel PJ, Poulter LW, Mackaness GB. Induction of cell-mediated immunity to Mycobacterium lepraemurium in susceptible mice. Infect Immun, 1977, 18, 654-659.

31 Lefford MJ, Mackaness GB. Suppression of immunity to Mycobacterium lepraemurium infection. Infect Immun, 1977, 18, 363-369. 